Cinémas

Revue d'études cinématographiques

Journal of Film Studies

\title{
Regard oblique, bifurcation et ricochet, ou de l'inquiétante étrangeté du carrello
}

\section{Elena Dagrada, André Gaudreault et Tom Gunning}

Volume 10, numéro 2-3, printemps 2000

Cinéma et intermédialité

URI : https://id.erudit.org/iderudit/024823ar

DOI : https://doi.org/10.7202/024823ar

Aller au sommaire du numéro

Éditeur(s)

Cinémas

ISSN

1181-6945 (imprimé)

1705-6500 (numérique)

Découvrir la revue

Citer cet article

Dagrada, E., Gaudreault, A. \& Gunning, T. (2000). Regard oblique, bifurcation et ricochet, ou de l’inquiétante étrangeté du carrello. Cinémas, 10(2-3), 207-223.

https://doi.org/10.7202/024823ar
Résumé de l'article

Le carrello, qui ponctue à maintes reprises le fameux film Cabiria de Giovanni Pastrone (1914), est l'une des figures les plus intrigantes du cinéma italien des années dix. Les historiens traditionnels du cinéma le voient généralement comme l'ancêtre du travelling. Tel ne serait pas le cas, selon les auteurs du présent article. Même s'il fait indubitablement partie de la famille des mouvements de caméra, même s'il implique un mouvement de la caméra sur un chariot, au même titre que le travelling institutionnel, le carrello cabirien serait doté de plusieurs traits spécifiques qui en font un cas unique dans l'histoire du cinéma. 


\title{
Regard oblique, bifurcation et ricochet, ou de l'inquiétante étrangeté du carrello
}

\section{Elena Dagrada, André Gaudreault et Tom Gunning}

\begin{abstract}
RÉSUMÉ
Le carrello, qui ponctue à maintes reprises le fameux film Cabiria de Giovanni Pastrone (1914), est l'une des figures les plus intrigantes du cinéma italien des années dix. Les historiens traditionnels du cinéma le voient généralement comme l'ancêtre du travelling. Tel ne serait pas le cas, selon les auteurs du présent article. Même s'il fait indubitablement partie de la famille des mouvements de caméra, même s'il implique un mouvement de la caméra sur un chariot, au même titre que le travelling institutionnel, le carrello cabirien serait doté de plusieurs traits spécifiques qui en font un cas unique dans l'histoire du cinéma.
\end{abstract}

\begin{abstract}
The carrello, that punctuates time and again Giovanni Pastrone's famous Cabiria (1914), is one of the most intriguing figures in Italian cinema of the 1910s. Traditional historians of cinema generally consider it as an ancestor of the traveling shot. Now, such would not be the case, according to the authors of this article. Even if it is indubitably part of the family of camera movements, and even if it implies the movement of a camera on a dolly, as in a traditional tracking shot, the "cabirian" carrello is endowed with several specific characteristics that make it a unique case in the history of cinema.
\end{abstract}


La recherche dont nous ferons état ici ${ }^{1}$ devait à l'origine porter exclusivement sur la confrontation entre le film Cabiria (Pastrone, 1914) et ses chutes, dont une bonne partie a été récemment retrouvée. Il devait y être essentiellement question de montage et de mise en scène. Mais en cours de route, cet étrange objet qu'est le carrello cabirien, objet aussi fabuleux que le film lui-même, s'est imposé à notre regard. C'est pourquoi nous avons dévié de notre itinéraire. Il s'agira ici de démontrer la spécificité du carrello et d'en tirer les conséquences, c'està-dire de situer Cabiria dans l'histoire du cinéma et de considérer la place essentielle qu'il y occupe.

Les historiens de l'ancienne génération ont beaucoup glosé sur le carrello cabirien, mais sans jamais proposer un schéma de lecture qui lui soit adéquat. Un chercheur de la nouvelle génération, Paolo Cherchi Usai, en a récemment proposé une interprétation d'un type nouveau dont Yuri Tsivian rend compte ainsi :

Cherchi Usai defined the "Cabiria movement" (or, rather, the "Cabiria-type movement") as a camera gesture that " invited" the viewer in the diegesis, that «introduced" him to the space of the film. Cherchi Usai calls this type of tracking shot a "come with me" camera movement " (p. 205-206).

Limpression première que donne le carrello cabirien est effectivement celle d'un emportement. On dirait que le mouvement de la caméra nous emporte avec lui, qu'il nous rapproche de la scène. Il faut dire aussi que le spectateur contemporain de la sortie de Cabiria a justement besoin de cette sorte d'invitation à entrer dans la diégèse, qu'il a nettement besoin d'encouragement à s'introduire, ne serait-ce que de façon "virtuelle ${ }^{2}$ », dans l'espace occupé par les personnages, de manière à se sentir plus intimement concerné par ce qui se passe à l'écran. Il n'est en effet pas loin ce temps où la confrontation exhibitionniste ${ }^{3}$ faisait la loi dans le monde des vues animées. Et le système d'intégration narrative qui allait justement favoriser une absorption diégétique dont l'institution avait besoin pour s'ériger ${ }^{4}$ n'est encore, en 1913-1914, qu'en voie de formation.

Il est possible, donc, de lire le carrello cabirien, du moins le carrello-in, comme une invitation faite au spectateur pour qu'il 
adopte la foulée et la direction du mouvement de la caméra; pour qu'il se déplace, lui aussi, ne serait-ce que symboliquement, en direction de ces ombres et figures qui sont projetées sur l'écran; pour qu'il essaie d'y trouver une place, pour qu'il essaie d'y faire sa place. Et pourtant, il semble qu'il faille considérer le carrello sous un autre angle, qu'il faille renverser l'orientation de sa trajectoire implicite. Certes, il serait bien ce mouvement qui amène le spectateur à se mobiliser, sur le plan symbolique, pour aller en direction des personnages de la diégèse et s'approcher d'eux; mais il serait encore, et dans le même temps, un mouvement qui amène les personnages de la diégèse en direction du spectateur, qui amène la scène elle-même à se rapprocher du spectateur : carrello-in, donc, mais aussi... plateau-in.

Il y a en tout cas un spectateur, et qui plus est un spectateur "professionnel ", qui, déjà en 1914, a considéré le carrello de pareille manière. Voici ce qu'écrit en effet un critique de cinéma dans le New York Dramatic Mirror:

Among the photographic novelties is one frequently used with fine effect. Scenes are slowly brought to the foreground or moved from side to side, quite as though they were being played on a movable stage. By this method full value is given to deep sets, and without any break the characters are brought close to the audience 5 .

"Les personnages sont amenés en direction des spectateurs [...]. " Ainsi, d'après ce critique contemporain, le carrello cabirien produirait un effet de rapprochement de l'instance actorielle en direction de l'instance spectatorielle plutôt que l'effet inverse, dont on sait par ailleurs qu'il est habituellement l'effet produit par le travelling dans le cinéma institutionnel. Comment expliquer cette disparité entre deux mouvements apparemment si semblables l'un à l'autre? C'est que le carrello cabirien et le travelling institutionnel (ou, mieux encore, le tracking institutionnel) ne relèvent pas du même paradigme et qu'ils ne s'adressent pas au même spectateur. Avec le tracking, c'est la caméra et avec elle le spectateur - qui se rapproche des objets ou des sujets filmés. Dans le cas du carrello, ce serait le contraire: ce seraient les objets filmés qui viendraient au-devant du spectateur, 
comme si on les lui servait sur un plateau... Alors que le travelling du cinéma classique permet à l'instance spectatorielle de se mobiliser pour s'introduire dans l'espace même de la représentation, un espace enveloppant, fondé précisément sur le centrement du spectateur, le carrello cabirien s'adresserait à un spectateur qui reste campé dans une position d'extériorité par rapport à cet impénétrable écran qui diffuse des images que son regard ne peut que frôler.

Voilà qui met en question la paternité attribuée au carrello. Ce dernier n'est peut-être pas ce que l'on a toujours dit de lui: l'ancêtre du travelling. Le carrello a beau être une sorte de travelling - vu la mobilisation effective de la caméra —, il doit en être distingué. Car il est tout le contraire du tracking qui, comme son nom l'indique, permet au cinéaste de traquer son ou ses personnages, telle une bête sa proie. Si le tracking frappe en plein dans l'cil, le carrello, lui, aussi tactile que visuel, ressemble plutôt à une caresse furtive, faite à fleur de peau et à l'aveuglette. Mais qu'est donc, au juste, le carrello cabirien? Désormais, il s'agit là pour nous d'une question essentielle, et à laquelle nous répondrons d'abord en abordant ce mouvement de caméra sous l'angle de ses fonctions, puis du point de vue de ses traits spécifiques.

Notre étude nous a permis de dégager trois fonctions du carrello cabirien, fonctions qui ne sont pas exclusives l'une de l'autre: 1 . le carrello peut avoir une fonction d'exploration; le mobile sur lequel on a monté la caméra amène celle-ci à circuler

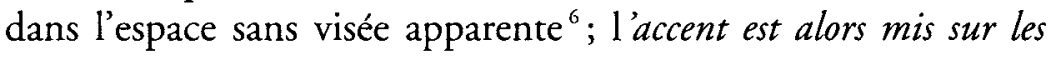
rapports spatiaux plutôt que sur le personnage ou sur le décor. 2. le carrello peut avoir une fonction d'indexation; il a comme visée apparente d'amener la caméra à mettre en relief action ou personnage; l'accent est alors mis sur le personnage plutôt que sur les rapports spatiaux ou sur le décor. 3. le carrello peut avoir une fonction de découverte; il a comme visée apparente d'élargir le champ de vision du spectateur, d'ouvrir la perspective du décor; l'accent est alors mis sur le décor plutôt que sur les rapports spatiaux ou sur le personnage.

Si ces fonctions s'apparentent à celles du tracking institutionnel, c'est dans leur modulation que la différence fondamentale 
entre le carrello cabirien et le tracking institutionnel est sensible. Cette modulation se produit en rapport avec les six traits qui fondent, selon nous, la spécificité du carrello, et que notre étude a permis de dégager. Ces caractéristiques spécifiques du carrello sont en rapport avec les divers paramètres de son existence, et chacune d'elles le distingue nettement du tracking institutionnel.

Tout d'abord, premier trait, le carrello circule le plus souvent de façon tangentielle par rapport à l'objet visé. La diagonale est sa trajectoire de prédilection; c'est sur elle que repose en grande partie la spécificité du carrello au sein de l'ensemble "mouvements de caméra". Le tracking institutionnel est un mouvement direct et frontal; le carrello est un mouvement double: à la fois travelling avant et travelling latéral, il est essentiellement ambigu. Quant au regard qu'il jette sur les choses, c'est un regard oblique, indirect, la caméra par laquelle il transite étant le plus souvent maintenue en position basse. Elle se tient non pas à hauteur d'homme, mais à hauteur de fauve, si l'on peut dire, à la hauteur d'un fauve qui ne traquerait pas ses victimes. La caméra mobile de Pastrone ne peut décidément pas affronter de plein fouet la scène qu'elle filme. Elle se place timidement en retrait de l'acteur, de biais et en contrebas. À l'instar du spectateur de l'époque, la caméra de Pastrone ne fait, elle aussi, que frôler la scène, sans pouvoir $\mathrm{y}$ pénétrer.

Mais dans sa trajectoire, et c'est là le deuxième trait qui fait sa spécificité, le carrello ne se limite jamais à une visée strictement narrative; il semble répondre à une logique qui lui est propre. Le carrello ne doit pas son existence à une volonté de mieux raconter les choses; la trajectoire qu'il emprunte n'est d'ailleurs généralement pas conçue en vue de la focalisation sur un moment particulier de l'action. Quand elle se mobilise, la caméra de Pastrone ne cible jamais un élément, un personnage ou un accessoire situés précisément dans l'aire de jeu. Le carrello observe plutôt les actions et les "acteurs", de loin, de ce regard indirect qui lui est si particulier. La raison d'être du carrello, c'est de donner au spectateur un sens des relations spatiales qui prévalent dans l'aire de jeu au sein de laquelle se déroulent les actions, comme si la motivation profonde du "glissement progressif" de la caméra et de sa trajectoire était précisément la

Regard oblique, bifurcation et ricochet, ou de l'inquiétante étrangeté du carrello 
modification même de cet espace, son changement de perspective. À l'inverse, le tracking institutionnel se présente habituellement comme le produit d'une conscience narratrice, comme le produit d'un narrateur qui sait où il va, qui a quelque chose à nous dire par son mouvement de caméra, et qui nous le dit. Le carrello cabirien laisse souvent l'impression qu'il n'est pas produit par une conscience, qu'il n'est pas ancré dans une instance, quelle qu'elle soit. Il laisse plusieurs questions ouvertes. Que veut-il me dire? Veut-il même me dire quelque chose? S'adresse-t-il à moi ? Le carrello cabirien est doté d'un très haut coefficient d'étrangeté. Il donne l'impression de n'avoir aucune visée, et ce, même quand il en a une.

Troisième trait, le carrello s'approche des personnages sans jamais s'immiscer dans leur espace intime; il franchit les distances, mais se garde à distance. Et s'il fait souvent se rapprocher instance actorielle et instance spectatorielle, ce rapprochement a des limites absolues. Jamais la caméra de Pastrone ne viole l'intimité de ses personnages par le truchement d'un carrello. Elle n'est autorisée à pénétrer dans l'espace intime du personnage que par l'intermédiaire d'une technique déjà considérée comme relativement violente: celle du cut-in (comme on l'a vu dans la première partie de la présente étude). C'est comme si la caméra de Pastrone jetait un regard oblique sur la scène qui se déroule de biais devant elle, et que ce regard bifurquait, ricochait sur cette scène, sans arriver à y pénétrer, sans arriver à la pénétrer. Comme si le monde profilmique, dans lequel cette scène se déroule, était séparé du monde filmographique ${ }^{7}$, dans lequel la caméra de Pastrone se tient, par un no man's land impénétrable pour la caméra et, partant, pour le spectateur. Alors que le gros plan du visage, dans le cinéma institutionnel, amène le spectateur à s'introduire dans l'espace intime du personnage et attire l'attention sur son intériorité, le carrello cabirien n'isole jamais, n'abstrait jamais complètement le personnage du décor dans lequel il agit. Certes, dans Cabiria, il arrive que le carrello nous rapproche un tant soit peu des personnages, mais plutôt que de nous impliquer émotivement, il nous maintient constamment à distance; il fait de nous des observateurs, des observateurs lointains comme dirait Burch ${ }^{8}$. 
Quant au rythme de croisière du carrello, il est, quatrième trait spécifique, d'une lenteur vertigineuse. Il franchit les distances, mais jamais avec ivresse. Si bien qu'il est souvent difficile de saisir le moment exact où la caméra se met en branle et le moment qui marque l'arrêt du mouvement. La lenteur est telle que, pour réussir à percevoir la mise en mouvement, l'analyste est tenu de détourner son regard du point focal de l'action pour surveiller attentivement les bords du cadre. C'est dire que le carrello cabirien est souvent à la limite du percept. Plutôt que d'avoir une conscience nette du mouvement, le spectateur a l'impression d'une sorte de frémissement qui traverse de part en part l'espace écranique, qui anime cet espace d'un étrange souffle de vie et le dote d'un pouvoir extrême de fascination: vertigineuse lenteur, à la limite du perceptible, qui est le propre du carrello cabirien.

Cinquième trait, le carrello est davantage attractionnel que narrationnel. Plutôt que de livrer des informations à des fins narratives, plutôt que de constituer un adjuvant de la narration, le carrello charme, intrigue l'œil. En effet, il relève du spectacle pour l'œeil, spectacle au sens faible du mot: «Ensemble de choses ou de faits qui s'offre au regard [...]" (Le Petit Robert). C'est comme cela, en tout cas, que l'a perçu un autre critique du New York Dramatic Mirror:

The scoffers came to see CABIRIA, and left overwhelmed with admiration, but only half-converted. «It is a triumph of pictorial art, they said, but its appeal is solely to the eye, and we have long granted that province to the motion picture? ${ }^{9}$."

Mais il y a plus. Quand on examine de près certains carrelli, on s'aperçoit qu'ils donnent carrément l'impression de la troisième dimension, produisant même un effet "stéréoscopique " assez remarquable, que Pastrone a déjà lui-même commenté ${ }^{10}$. Cet effet stéréoscopique a des conséquences sur la perception de l'instance spectatorielle: il lui semble alors que l'espace se met à vibrer, à s'animer, comme si le carrello lui avait insufflé la vie... Par ailleurs, le mouvement de translation caractéristique du carrello cabirien donne l'impression que les rapports entre les objets

Regard oblique, bifurcation et ricochet, ou de l'inquiétante étrangeté du carrello 
et les personnages sont aussi palpables, sinon plus, que les personnages et les objets eux-mêmes. Pastrone fait, en somme, une mise en scène qui s'intéresse à l'espace entre les choses autant et sinon plus qu'aux choses elles-mêmes, un espace en négatif que ses mouvements de caméra rendent visible.

Finalement, sixième et dernier trait, le carrello se laisse percevoir par le spectateur sans pour autant attirer l'attention sur lui : spectacle pour l'œil, certes, mais spectacle non spectaculaire. Le carrello de Pastrone est assez puissant pour, littéralement, animer l'espace, mais il le fait sans jamais attirer l'attention sur le dispositif même de mobilisation de la caméra. Si bien qu'on ne le remarque, lui-même, pas tellement... C'est qu'il relève d'une technique subtile, qui semble vouloir se faire oublier, qui semble vouloir faire oublier sa nature proprement machinique, qui semble vouloir faire oublier l'insoutenable lourdeur du dispositif technique à qui il doit pourtant son existence. On dirait que le carrello s'avance à pas feutrés sur la scène, sur cette autre scène, sans vouloir se faire remarquer, sans vouloir se faire remarquer des spectateurs, bien sûr, non plus que des acteurs.

Ainsi, le carrello cabirien serait une structure apte à troubler le spectateur, en raison de sa nature essentiellement ambiguë et contradictoire. D'un côté, il produit un effet de réalité, par le relief, par le volume qu'il donne à la représentation spatiale. D'un autre côté, cependant, il produit un inéluctable sentiment d'étrangeté, une inquiétante étrangeté de l'ordre de l'Unheimliche freudien, en ce qu'il vient déstabiliser l'espace représenté, en l'animant d'un mystérieux mouvement, qui vient de nulle part et qui semble n'aller nulle part. Le spectateur se souvient moins des nouvelles perspectives spatiales auxquelles le carrello lui donne accès que de cette instabilité quasi imperceptible communiquée à l'espace par la mobilisation de la caméra, une instabilité susceptible de provoquer chez lui un doux vertige, sur la base d'une expérimentation inédite, et virtuellement étourdissante, des relations spatiales. Le carrello arrive de biais, de façon tangentielle, sans crier gare, sans s'annoncer, sur le bout des pieds, en catimini, comme pour ne pas déranger les comédiens - à moins qu'il ne s'agisse des personnages? C'est que le carrello n'est pas seulement un procédé sublime, c'est aussi, et surtout, un procédé subliminal. 
La particularité du carrello, son unicité, exige de le situer dans l'histoire du cinéma. L'usage qui est fait du mouvement de caméra dans Cabiria est représentatif d'une certaine tendance proprement européenne du cinéma des années dix, qui privilégie la composition spatiale en profondeur, par opposition à cette autre tendance, alors dominante aux États-Unis, qui privilégie un montage segmentant l'espace de la représentation. Ainsi le carrello cabirien se situe-t-il dans le prolongement d'une conception esthétique qui donne la préférence aux procédés de mise en cadre (mouvements de caméra, composition en profondeur, etc.) voire à un découpage visuel interne au plan (un "découpage" sans montage, donc) - plutôt qu'aux procédés de mise en chaîne (agglomération et juxtaposition de segments spatio-temporels).

Mais en raison des caractéristiques que nous avons fait ressortir, les mouvements de caméra de Cabiria excèdent le type de mise en scène axée sur la composition et le jeu en profondeur qui domine alors en Europe. D'ailleurs, ils ne seront pas adoptés par la confrérie des cinéastes, à la différence du type de mouvements de caméra dont les Bauer, Griffith et autres Tourneur orneront leurs films. À titre de travelling, car c'en est un, le carrello de Cabiria est une figure que l'on peut certes rapporter au processus de narrativisation du cinéma, mais c'est une figure qui fait preuve d'un trop-plein, qui déborde des limites acceptables de la configuration "classique", un peu comme cette autre figure de l'excès qu'est le montage parallèle d'Intolerance, qui restera, lui aussi, un cas relativement unique dans les annales du cinéma plutôt qu'un modèle à suivre. Cabiria comme Intolerance recèlent quelque chose des œuvres d'avant-garde. Ils sont en excès par rapport aux normes de la période de leur production mais, au contraire des ceuvres d'avant-garde stricto sensu, ce n'est pas délibérément que ces normes sont mises en question.

Les excès de Cabiria sont liés à la volonté de résoudre le problème crucial, à l'époque de sa production, du rapport du spectateur avec la scène représentée sur l'écran, de l'appréhension spectatorielle de l'espace mis en scène. Et la réponse de Pastrone, c'est de privilégier, au moyen du carrello, la mobilité du regard spectatoriel tout en préservant la continuité spatiale et temporelle. Plus précisément, la réponse de Cabiria, c'est de mobiliser 
l'espace scénique dans lequel l'action a lieu, en accentuant son aspect tridimensionnel, son épaisseur, tout en maintenant le spectateur dans les marges de la scène. C'est la raison pour laquelle on peut avoir le sentiment, devant Cabiria, que le film met l'accent sur la représentation, non pas d'un drame, mais d'une aire de jeu. Voilà qui explique, croyons-nous, la relative indifférence de la caméra, dans le carrello, par rapport au drame humain qui se joue dans l'espace qu'il a la charge de représenter. Il y a quelque chose dans la mécanique bien huilée du carrello cabirien qui rappelle La Région centrale (1971) de Michael Snow.

Plusieurs des carrelli de Pastrone pourraient servir à démontrer l'à-propos de l'hypothèse que nous avançons ici. Mais il en est un, le plus fabuleux de tous, qui mérite une attention particulière. Il s'agit du long mouvement de caméra à la faveur duquel nous voyons Maciste explorer la cave à l'intérieur de laquelle Fulvio et lui sont prisonniers (voir les photos 1 à 6 ). Ce carrello est vraiment exemplaire pour la réflexion que nous proposons ici, car il rassemble en lui l'ensemble des fonctions et traits spécifiques du carrello tels que nous les avons définis, et qu'il offre de surcroît une figure de mise en abyme particulièrement excessive et remarquable.

Cette mise en abyme prend la forme d'un gag de Maciste à l'endroit de Fulvius; à moins qu'il ne s'agisse d'un gag de Pastrone à notre intention, par Maciste et Fulvio interposés. Quoi qu'il en soit, cette blague est l'occasion d'un carrello particulièrement propice à la production d'effets stéréoscopiques, en raison de l'arabesque de son déplacement, particulièrement sinueux ${ }^{11}$, dans un décor tout en profondeur et dont le principal élément consiste en une série d'amphores étalées dans la profondeur, par étages successifs. Le mouvement culmine sur ce geste de Maciste faisant mine de boire à même une amphore, bidimensionnelle celle-là, dessinée à la craie sur un mur par Fulvio. Une amphore qui apparaît dans ce qu'il est indiqué de considérer comme la mise en abyme de l'effet visuel produit par le carrello cabirien, et qui pointe effectivement la nature tridimensionnelle caractéristique de l'effet produit par le mouvement cabirien. Une amphore qui est une "métaphore" - une méta(m)phore? - , une cita- 


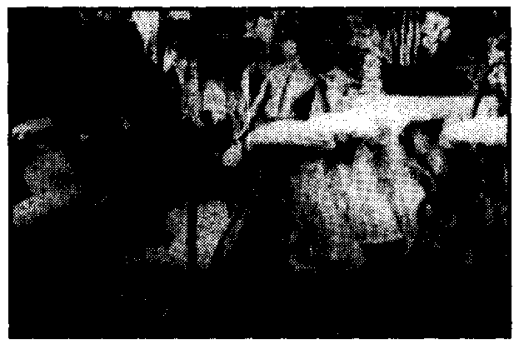

Photo ${ }^{0} 1$

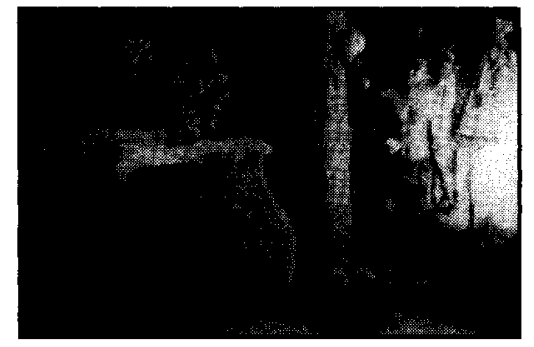

Photo $\mathrm{n}^{\circ} 2$

Cabiria (Giovanni Pastrone, 1914)

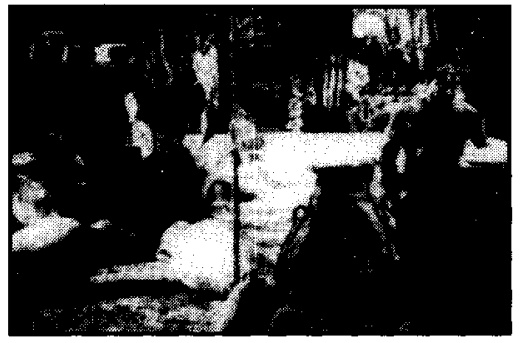

Photo $^{\circ} 3$

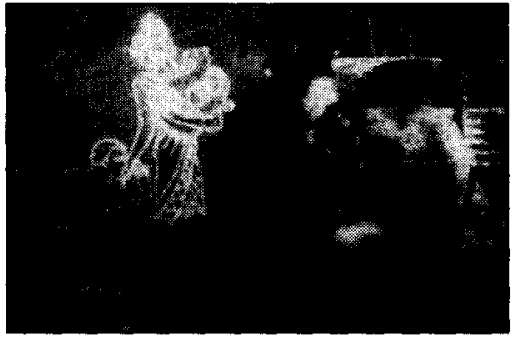

Photo $n^{\circ} 4$

Cabiria (Giovanni Pastrone, 1914)

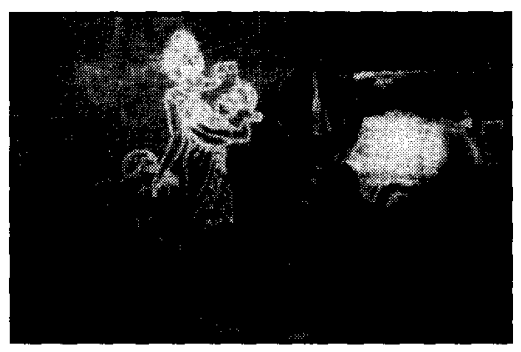

Photo $\mathbf{n}^{\circ} 5$

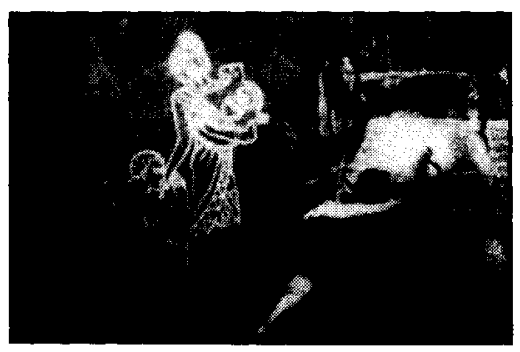

Photo $n^{0} 6$

Cabiria (Giovanni Pastrone, 1914)

tion bidimensionnelle des amphores tridimensionnelles, en "dur", qui figurent dans le décor et qui auraient eu droit, elles aussi, à un traitement bidimensionnel, n’eût été ce plus-queperspectiviste carrello, cet ultra-stéréoscopique carrello par le truchement duquel elles nous sont montrées ${ }^{12}$.

Regard oblique, bifurcation et ricochet, ou de l'inquiétante étrangeté du carrello 
Un autre carrello de Pastrone, cette fois dans Tigre Reale (1916), présente une mise en abyme aussi métaphorique que celle que nous venons de décrire. Il s'agit d'un carrello fort édifiant, qui ouvre sur une perspective à couper le souffle et qui fait glisser le point de vue de la caméra de l'acteur, sur scène, au spectateur, dans la salle. Ce mouvement, on peut le lire, croyonsnous, comme une métaphore de l'intention qui est au fondement même du mouvement cabirien, soit celle d'essayer de mettre en rapport, par un heureux effet de suture, l'instance actorielle avec l'instance spectatorielle (voir les photos 7 et 8).

Comment ne pas lire dans ce fragment l'insistante question, posée au spectateur, sur la nécessaire tâche du cinéaste de faire passer un courant entre la scène représentée et les spectateurs qui lui font face? Comment ne pas lire ce carrello comme une volonté de poser la question, cruciale en ces années-là, de la suture à opérer entre l'instance actorielle et l'instance spectatorielle, et en même temps, comme une volonté de les emporter toutes deux dans le même... mouvement? Un mouvement qui est redoublé, sur le plan filmographique, par le mouvement même de la caméra de Pastrone, dont notre recherche aura, nous l'espérons, permis de percer quelques secrets.

La question de la suture entre instance actorielle et instance spectatorielle est également au centre de la dichotomie que nous avons évoquée plusieurs fois, celle qui oppose le montage et la composition spatiale en profondeur. Cette question est au centre du choix paradigmatique qui semble se dessiner durant la première moitié des années dix, et qui oppose cette tendance plutôt européenne à l'analyse de l'espace à travers la composition en profondeur à cette autre tendance qui privilégie l'analyse de l'espace à travers sa segmentation, tendance que l'on retrouve plutôt aux États-Unis. Il s'agit là d'une opposition qui revient souvent, tant dans l'histoire du cinéma que dans celle des théories du cinéma. Elle est parfois formulée comme une opposition entre l'ancien et le nouveau, d'autres fois comme une opposition entre le "réalisme" et le "symbolisme", ou entre le "classicisme" et la "modernité». Et d'autres fois encore, pour reprendre l'heureuse formule d'André Bazin ${ }^{13}$, elle consiste à opposer les cinéastes qui croient à l'image et ceux qui croient en la réalité... 


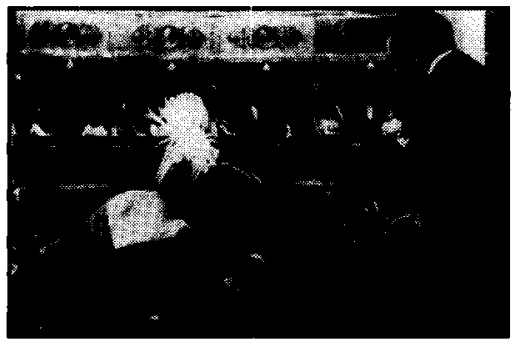

Photo $\mathbf{n}^{\circ} 7$

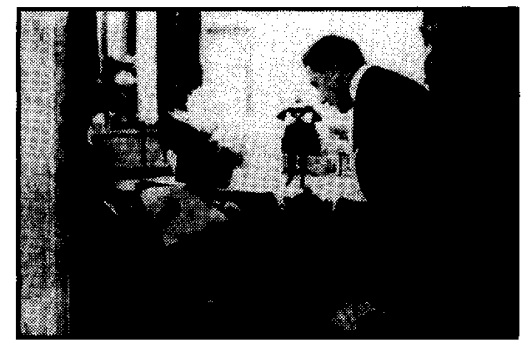

Photo $\mathrm{n}^{\circ} 8$

Cabiria (Giovanni Pastrone, 1914)

Malgré ce que les deux tendances peuvent avoir de commun, il s'agit là d'une opposition dont les contours apparaissent clairement comme le fruit de deux esthétiques distinctes. L'étude de $\mathrm{Ca}$ biria nous aide non seulement à comprendre cela, mais aussi ce qui rapproche les uns des autres les films qui s'inscrivent dans cette tradition trop souvent méconnue, même aujourd'hui, que représente la tendance européenne de la première moitié des années dix à privilégier la composition en profondeur plutôt que le montage.

Le montage a revêtu une importance de premier plan à partir de la fin des années dix et tout au long des années vingt, à la fois pour le cinéma classique qui commence à émerger à Hollywood et pour le cinéma des "avant-gardes", qui prend son envol en France et en Union soviétique. Au sein des avant-gardes, on a même assisté à l'élaboration d'une production théorique qui voyait dans le montage la quintessence même du cinéma et dans les figures comme le gros plan ou les effets optiques, le chemin à suivre pour faire du cinéma un art. Tant et si bien que cette première tendance à la composition spatiale en profondeur s'est en quelque sorte éclipsée des mémoires.

Théoriciens et cinéastes ne se gênaient pas, à cette époque, pour avancer que l'absence de montage dans un film lui conférait des attributs spécifiquement théâtraux et en faisait une œuvre arriérée et dépassée. Le recours à la théâtralité était pourtant loin de faire partie du programme esthétique des cinéastes fidèles à la tradition européenne, qui misait sur la composition en profondeur et dont Cabiria est un digne représentant malgré l'excès de ses carrelli. Cette tradition est en effet loin de se 
fonder sur une esthétique théâtrale, encore moins sur une esthétique "réaliste" du type de celle que théorisera Bazin, dans les années cinquante, en la définissant à partir du plan d'ensemble et de la profondeur de champ. Elle se base plutôt sur l'idée d'unicité, de spécificité de l'espace scénique interne à l'espace filmique, ainsi que sur la volonté de souligner pareille unicité dans son rapport au spectateur.

La composition spatiale en profondeur, telle qu'elle s'est présentée dans le cinéma européen de la première moitié des années dix, aspire en fait à la création d'une relation unique et privilégiée de chaque spectateur avec la scène. Elle tend à opérer, entre instance actorielle et instance spectatorielle, une suture qui tienne compte de cette relation unique et privilégiée. Et, comme l'a montré Yuri Tsivian dans ses réflexions sur Bauer, elle se fonde précisément sur les caractères différentiels de l'espace scénique au cinéma et au théâtre. Au contraire du théâtre, où chacun des spectateurs accède à un point de vue qui lui est propre sur la scène se déroulant devant lui, le cinéma impose à tous les spectateurs le même point de vue, la même et unique perspective; en cela, le cinéma est plus proche de la peinture, qui peut se permettre une composition d'une précision dont le théâtre est privé. Au contraire de la peinture, cependant, le cinéma possède les attributs du mouvement ; le cinéaste peut non seulement distribuer les acteurs de façon soignée dans la perspective de son "tableau», mais il peut également les faire bouger de manière à diriger l'attention du spectateur sur ce qui compte le plus dans l'action dépeinte. C'est ainsi qu'ont travaillé les Sjöström, Blöm, Bauer et Feuillade, qui utilisaient la composition en profondeur et le mouvement des acteurs (quand ce n'est pas le mouvement de la caméra) pour mettre en relief les moments les plus importants sur le plan narratif, sans avoir recours de façon systématique à la fragmentation de l'espace. C'est ainsi que travaille aussi Pastrone, qui "compose" ses scènes, tout en les "mobilisant" par le truchement des déplacements de ses acteurs. Ou encore, en ayant recours au carrello; c'est alors l'espace scénique luimême qui est mobilisé en direction du spectateur, lui offrant ainsi une représentation tridimensionnelle au sein de laquelle, aussi actif que distant, il reste captivé par des rapports perspec- 
tifs toujours nouveaux, dans les marges desquelles il est condamné à demeurer.

L'étude de Cabiria, de même que la confrontation du produit fini avec ses chutes, confirme tout cela, et elle montre jusqu'à quel point la conception de ce film est le fruit d'une véritable recherche sur l'espace. Elle montre aussi toutes les capacités de ce film eu égard à la maitrise des différentes figures de montage déjà élaborées à l'époque de sa réalisation. Ainsi Cabiria n'est-il pas un film qui serait, encore et toujours "primitif", ce n'est pas non plus un film situé quelque part entre l'ancien et le nouveau. Au contraire, même s'il s'agit, à l'instar de ses carrelli, d'une œuvre unique, Cabiria est un film pleinement "de son temps ${ }^{14}{ }$. C'est en tout cas ce que nous démontrent la présence, en son sein, de certaines figures de montage et son approche toute particulière de la composition spatiale en profondeur.

\section{Université de Bordeaux 3, Université de Montréal et University of Chicago}

\section{NOTES}

1 Voir la première partie du présent texte dans le dernier numéro de Cinémas ("Composition en profondeur, mobilité et montage dans Cabiria", vol. X, $\mathrm{n}^{\circ} 1$, automne 1999, p. 55-78). Le texte complet est paru initialement en italien sous le titre "Lo spazio mobile. Del montaggio c del carrello in Cabiria", dans Paolo Bertetto e Gianni Rondolino (a cura di), Cabiria e il suo tempo (Torino/Milano: Museo Nazionale del Cinema/Edirrice Il Castoro, 1998, p. 150-183). Nous remercions les éditeurs d'avoir permis sa parution en français. Par ailleurs, rappelons ici que cette recherche a été réalisée en collaboration avec le GRAFICS (Groupe de recherche sur l'avènement et la formation des institutions cinématographique et scénique) de l'Université de Montréal, subventiorné par le Conseil de recherches en sciences humaines du Canada et par le Fonds FCAR du Québec. La contribution d'André Gaudreault a été réalisée dans le cadre d'une recherche sur l'avènement et le développement du montage, rendue possible grâce à une Bourse Killam (1997-1999) attribuée par le Conseil des Arts du Canada. Les auteurs remeicient Stéphanie Côté, du GRAFICS, pour son support technique; Paolo Bertetto, du Museo Nazionale del Cinema de Turin, pour sa généreuse disponibilité ; Claudia Gianetto et Alessandra Torrione, du Museo Nazionale del Cinema de Turin, pour leur diligence.

$2 \AA$ ce sujet, voir les travaux de Jean-Pierre Sirois-Trahan, qui oppose regard "virtuel " et regard "propre": "La théâtralité chez Méliès et le regard du spectateur du cinéma des premiers temps" (communication au Congrès mondial de l'Association internationale du théâtre à l'université, Université Laval, juin 1997, à paraître); 
"Réception des nouvelles images et cinéma des premiers temps " (Sociétés \& Représentations, "La croisée des médias", $n^{\circ}$ 9, 2000, p. 143-160).

3 Sur les notions de confrontation exhibitionniste, d'absorption diégétique et de système d'intégration narrative, voir l'ouvrage de Tom Gunning intitulé $D$. W. Griffith and the Origins of American Narrative Film (Urbana and Chicago: University of Illinois Press, 1991), celui d'André Gaudreault, Du littéraire au filmique. Système du récit (Paris/Québec: Armand Colin/Nota bene, 1999) ainsi que l'article signé par André Gaudreault et Tom Gunning et intitulé "Le cinéma des premiers temps: un défi à l'histoire du cinéma?" (dans Jacques Aumont, André Gaudreault et Michel Marie (direction), Histoire du cinéma. Nouvelles approches, Paris: Publications de la Sorbonne, 1989, p. 49-63).

4 L'«institution ” dont il est ici question est celle du cinéma classique de type hollywoodien.

5 New York Dramatic Mirror, 13 mai 1914, p. 40. Cité par George Pratt dans son ouvrage intitule Spellbound in Darkness: A History of the Silent Film (Greenwich: New York Graphic Society, 1973, p. 126); cité également (mais en partie seulement) par Yuri Tsivian dans Early Cinema in Russia and Its Cultural Reception (London : Routledge, 1994, p. 209).

6 Comme si la caméra restait non ciblée, "untargeted", pour reprendre l'expression que David Bordwell utilisa en 1990 au cours d'une conversation avec Tom Gunning à propos des mouvements de caméra dans Cabiria.

7 À propos de la distinction entre "profilmique " et "filmographique », voir $D u$ littéraire au filmique d'André Gaudreault (Paris/Québec: Armand Colin/Nota bene, 1999).

8 Nous faisons ici allusion au titre d'un ouvrage de Noël Burch, Pour un observateur lointain. Forme et signification dans le cinéma japonais (Paris: Cahiers du cinéma/Gallimard, 1982).

9 New York Dramatic Mirror, 10 mars 1915, p. 28; cité par Pratt dans Spellbound in Darkness: A History of the Silent Film (Greenwich: New York Graphic Society, 1973, p. 206).

10 À ce sujet, voir l'article de Georges Sadoul intitulé "La tecnica rivoluzionaria nella Cabiria di Pastrone" (Cinema, n.s., a. IV, n. 58, 1951) et celui de Mario Verdone intitulé "Pastrone, ultimo incontro" (Bianco e Nero, a. XXII, n. 1, gennaio 1961). Signalons aussi une critique désapprobatrice de Pier da Castello parue dans $I l$ Maggese Cinematografico (Turin, 25-4-1914) et que cite Vittorio Martinelli dans $l l$ cinema muto italiano. 1914 (Bianco e Nero, année LIII, n. 1, Biblioteca di Bianco e Nero, 1993. p. 78-79).

11 Ce carrello a retrouvé sa longueur originelle lors de la restauration de 1995 (pour connaître les détails de ce travail de restauration, on pourra se reporter à la note 3 de la première partie de la présente étude). Dans les autres copies en circulation, il était plus court et beaucoup moins sinuosoïdal. Toute la première partie du mouvement, de gauche à droite, était en fait manquante. Pastrone avait dû lui-même, à l'époque, juger excessif un pareil mouvement de caméra...

12 Yuri Tsivian, avec lequel les auteurs de la présente communication ont discuté de leurs hypothèses sur Cabiria, avait lui aussi remarqué le sens métaphorique de cette fameuse scène. Les conclusions que nous tirons ici ont pu avantageusement bénéficier de la discussion en question.

13 Bazin formule cette idée dans "Lévolution du langage cinématographique" (Qu'est-ce que le cinéma?, Paris: Cerf, 1958, p. 131-148).

14 Sur la nécessité de reconnaitre au cinéma des années dix un statut spécifique, voir les travaux d'Éric De Kuyper : "Le cinéma de la seconde époque : le muet des an- 
nées dix" (Cinémathèque, $\mathrm{n}^{\circ} 1,1992$ ) et "Le cinéma de la seconde époque: le muet des années dix " (Cinémathèque, $\mathrm{n}^{\circ} 2,1992$ ).

\section{OUVRAGES CITÉS}

Bazin, André. Qu'est-ce que le cinéma? Paris : Cerf, 1958.

Burch, Noël. Pour un observateur lointain. Forme et signification dans le cinéma japonais. Paris: Cahiers du cinéma/Gallimard, 1982.

Pratt, George. Spellbound in Darkness: A History of the Silent Film. Greenwich : New York Graphic Society, 1973.

Tsivian, Yuri. Early Cinema in Russia and Its Cultural Reception. London: Routledge, 1994, p. 205-206. 